\title{
Estudo da cinética de secagem da pimenta de cheiro em diferentes temperaturas
}

\author{
Study smell pepper drying kinetics in different temperature
}

\author{
João C. S. Melo ${ }^{1}$, Emanoel D. Pereira ${ }^{2}$, Keville P. Oliveira ${ }^{3}$, Carlos H. C. Costa ${ }^{4}$ Regilane M. Feitosa ${ }^{5}$
}

Resumo: A pimenta de cheiro é um produto natural e vem sendo alvo de diversos estudos, em razão de apresentar propriedades importantes para a saúde e aprimorar sabores aos alimentos. Estudos direcionados vem chamando a atenção e um meio de conservação é de suma importância para que possa ser difundido pelo mundo. A secagem é um método de conservação muito utilizado para diminuir o teor de água do alimento, visando prolongar a sua vida útil durante o armazenamento. Diversos modelos matemáticos têm sido utilizados para descrever o processo de secagem. Este trabalho objetivou ajustar modelos matemáticos de secagem em camada fina, aos dados experimentais obtidos na secagem da pimenta de cheiro em diferentes temperaturas de secagem $\left(50,60\right.$ e $\left.70{ }^{\circ} \mathrm{C}\right)$, verificar a difusividade efetiva e a energia de ativação. Os resultados da secagem foram avaliados ajustando-se aos modelos: Newton, Page, Henderson \& Pabis, Logaritmico, Dois termos, Wang e Sing, Midilli et al., Henderson \& Pabis modificado e de Fick. A maioria dos modelos utilizados apresentou coeficientes de determinação maiores que 0,98, descrevendo satisfatoriamente a secagem do produto. A difusividade efetiva aumentou com a elevação da temperatura de secagem e o valor da energia de ativação foi de $38,66 \mathrm{~kJ} \mathrm{~mol}^{-1}$.

Palavras-chaves: Capsicum, difusividade, energia de ativação, modelagem matemática

\begin{abstract}
The hot peppers is a natural product and has been the target of several studies due to present important properties for health and enhance flavors to food. Directed studies has attracted the attention and a means of conservation is very important for it to be widespread throughout the world. Drying is a preservation method widely used to reduce the water content of the food, aiming extend its useful life during storage. Several mathematical models have been used to describe the drying process. This study aimed to adjust mathematical models of drying thin layer, the experimental data obtained in hot peppers drying in different drying temperatures $\left(50,60\right.$ and $\left.70{ }^{\circ} \mathrm{C}\right)$, check the effective diffusivity and activation energy. The results of drying were evaluated by adjusting to the models : Newton, Page , Henderson \& Pabis, logarithmic, Two terms , and Wang Sing , Midilli et al, Henderson and modified Pabis and Fick. Most models used had correlation coefficients greater than 0.98 , depicting satisfactorily drying. The effective diffusivity increased with drying temperature elevation and the value of the activation energy was $38.66 \mathrm{~kJ} \mathrm{~mol}^{-1}$.
\end{abstract}

Key words: Capsicum, diffusivity, activation energy , mathematical modeling

\footnotetext{
*Autor para correspondência

Recebido para publicação em 05/01/2015; aprovado em 25/05/2015

${ }^{1}$ Químico industrial, Dr. Técnico de Laboratório, IFRN. Caicó - RN.E-mail: jcarlosmequi@yahoo.com.br

${ }^{2}$ Técnico, Discente, Instituto Federal do Rio Grande do Norte, IFRN. E-mail: Emanuel.dantas@academico.ifrn.edu.br

${ }^{3}$ Químico, Técnico de Laboratório, IFRN. E-mail: keville.oliveira@ifrn.edu.br

${ }^{4}$ Tecnólogo de Alimentos, Especialista, Prof. Instituto Federal do Rio Grande do Norte, IFRN. E-mail: carlos.chaves@ifrn.edu.br

${ }^{5}$ Tecnóloga de Alimentos, Pesquisadora PNPD/CAPES, Engenharia de Processos, UFCG Campina Grande, PB. E-mail: regilanemarques@yahoo.com.br
} 


\section{INTRODUÇÃO}

A pimenta de cheiro é a mais brasileira de todas as espécies de pimenta domesticada e a região amazônica é a que concentra a maior diversidade (ARAÚJO et al., 2013). Caracterizada como uma hortaliça pertencente à família Solanaceae e ao gênero Capsicum L., o Brasil é conhecido como centro secundário dessa espécie, e os indígenas da região amazônica são os responsáveis pela sua domesticação (CERQUEIRA, 2012). Sendo que a produção de pimentas vem crescendo consideravelmente no Brasil, principalmente nos estados de Minas Gerais, São Paulo, Goiás, Ceará e Rio Grande do Sul (EMBRAPA, 2007).

O gênero Capsicum apresenta grande versatilidade, desde a alimentação até na perfumaria. Compõe uma importante parte do mercado de hortaliças frescas do Brasil, e também do segmento de condimentos, temperos e conservas, a nível mundial, em razão de dispor de meios que possibilitem o seu uso na forma fresca ou seca, inteira ou em pó, e até combinada com outros aromatizantes (DUTRA et al., 2010).

Além do fator comercial, a pimenta de cheiro vem sendo utilizado cada vez mais no nosso dia-a-dia, principalmente pelo motivo de ter características importantes que dá qualidade aos alimentos, como: aroma marcante, sabor picante e além de possuir propriedades medicinais comprovadas como atividade antioxidante e anticancerígeno que traz benefícios a saúde do ser humano (SANTOS et al., 2012). As pimentas doces e picantes podem ser processadas na forma de pó, flocos, picles, escabeches, molhos líquidos, conservas de frutos inteiros, geleias, entre outros (REIS et al., 2011).

A secagem é uma forma de conservação, que consiste em reduzir o teor de água do produto e, consequentemente, a sua atividade de água, com o objetivo de inibir o crescimento microbiano responsável pela deterioração, além de facilitar o transporte e o armazenamento do produto seco (GAVA,1984).

Segundo Afonso Júnior \& Corrêa (1999), o estudo de sistemas de secagem, seu dimensionamento, otimização e a determinação da viabilidade de sua aplicação comercial, podem ser feitos por simulação matemática, cujo princípio se fundamenta na secagem de sucessivas camadas delgadas do produto, utiliza-se um modelo matemático que representa satisfatoriamente a perda de umidade do produto durante o período de secagem. Vários modelos matemáticos que descrevem o mecanismo de secagem têm sido sugeridos para a otimização do processo de secagem e no desenvolvimento eficaz de projeto de secadores (DARVISHI et al., 2014).

Estes modelos, geralmente, baseiam-se em variáveis externas ao produto, como a temperatura e a umidade relativa do ar de secagem; porém, não fornecem indicações sobre os fenômenos de transporte de energia e de água no interior dos produtos e consideram que todo o processo de secagem ocorre somente no período de taxa decrescente (RESENDE et al., 2010).

O uso de modelos matemáticos para prever o processo de secagem de algumas pimentas vem sendo o objeto de vários estudos: pimenta de cheiro (PONTES et al., 2009), pimenta malagueta (SANTOS et al., 2012), pimenta Cambuci (DERLAN et al., 2013), pimenta verde (DARVISHI et al., 2014), entre outras.

Portanto, esse trabalho objetivou-se determinar as curvas e o tempo de secagem da pimenta e ajustar os modelos matemáticos de secagem em camada fina aos dados experimentais obtidos na secagem da pimenta de cheiro em diferentes temperaturas de secagem, definindo o melhor modelo matemático, verificar sua difusividade efetiva e a energia de ativação.

\section{MATERIAL E MÉTODOS}

A pimenta de cheiro foi adquirida na feira central da cidade de Caicó/RN e foi selecionada e triturada no laboratório de química do Instituto Federal do Rio Grande do Norte na cidade de Caicó/RN. As secagens foram realizadas com três repetições, utilizando-se a estufa, sem circulação de ar, nas temperaturas de 50,60 e $70^{\circ} \mathrm{C}$.

As curvas de secagem foram ajustadas aos dados experimentais utilizando-se nove diferentes equações empíricas e semi-empíricas, descrita na Tabela 1.

Tabela 1 - Modelos matemáticos utilizados para descrever a secagem da pimenta de cheiro Modelos matemáticos Equações

\begin{tabular}{ll}
\hline Page & $R U=\operatorname{Exp}\left(-k \cdot t^{n}\right)$ \\
Dois termos & $R U=a \cdot \operatorname{Exp}\left(-k_{0} \cdot t\right)+b \cdot \operatorname{Exp}\left(-k_{1} \cdot t\right)$ \\
Newton & $R U=\operatorname{Exp}(-k \cdot t)$ \\
Henderson e Pabis modificado & $R U=a \cdot \operatorname{Exp}(-k \cdot t)+b \cdot \operatorname{Exp}\left(-k_{0} \cdot t\right)+c \cdot \operatorname{Exp}\left(-k_{1} \cdot t\right)$ \\
Henderson e Pabis & $R U=a \cdot \operatorname{Exp}(-k \cdot t)$ \\
Logarítmico & $R U=a \cdot \operatorname{Exp}(-k \cdot t)+c$ \\
Midilli et al. & $R U=a \cdot \operatorname{Exp}\left(-k \cdot t^{n}\right)+b \cdot t$ \\
Difusão três termos & $R U=\frac{8}{\pi^{2}} \sum_{o}^{\infty} \frac{1}{(2 n+1)^{2}} \operatorname{Exp}\left(-(2 n-1)^{2} \pi^{2} D \frac{t}{4 L 2}\right)$ \\
Wong e Sing & $R U=1+a \cdot t+b \cdot t^{2}$ \\
\hline
\end{tabular}

Em que: RU - razão de umidade do produto, adimensional; $\mathrm{t}$ - tempo de secagem, h; $\mathrm{k}$ - coeficientes de secagem, a, b, $\mathrm{n}$ - constantes dos modelos. 
O ajuste dos modelos foi realizado pelo método QuasiNewton por meio de análises de regressão não linear. O grau de ajuste de cada modelo foi considerado pela magnitude do coeficiente de determinação $\left(R^{2}\right)$ e o menor erro médio estimado (SE).

Calculou-se a razão de umidade (RU) do produto para o tempo t, conforme apresentado na Equação 1.

$$
R U=\frac{U-U e}{U i-U e}
$$

Sendo: $\mathrm{U}$ - teor de água do produto no tempo, \%b.s;

$\mathrm{U}_{\mathrm{e}}$ - umidade de equilíbrio, \%b.s;

$\mathrm{U}_{0}-$ teor de água inicial do produto, \%b.s

O erro médio estimado para cada modelo foi calculado conforme demonstrado na Equação 2.

(2)

$$
S E=\sqrt{\frac{\sum\left(Y-Y_{0}\right)^{2}}{G L R}}
$$

Sendo: $\mathrm{n}$ - número de observações;

$\mathrm{Y}$ - valor observado;

$\mathrm{Y}_{0}$ - valor estimado pelo modelo;

GLM - graus de liberdade do modelo

A dependência da difusividade efetiva (D) com a temperatura foi analisada pela equação de Arrhenius:

$$
\mathrm{D}=\mathrm{A} \exp \left(-\frac{\mathrm{E}}{\mathrm{RT}}\right)
$$

Onde: $\mathrm{A}$ - constante, $\mathrm{m}^{2} \mathrm{~s}^{-1}$;

$\mathrm{E}$ - energia de ativação, $\mathrm{J}^{\mathrm{mol}^{-1}}$;

$\mathrm{R}$ - constante universal dos gases, 8,314 $\mathrm{J} \mathrm{mol}^{-1} \mathrm{~K}^{-1}$;

$\mathrm{T}$ - temperatura absoluta, $\mathrm{K}$

\section{RESULTADOS}

Na Figura 1 têm-se as cinéticas de secagem em camada fina da pimenta de cheiro em diferentes temperaturas. Nota-se que o aumento da temperatura $\left(50,60\right.$ e $\left.70^{\circ} \mathrm{C}\right)$ do ar de secagem reduziu o tempo de secagem da pimenta, cujos tempos foram iguais a 540, 490 e $400 \mathrm{~min}$, respectivamente. Pontes et al. (2009) estudando a pimenta de cheiro, nas temperaturas de secagem iguais a 50,60 e $70^{\circ} \mathrm{C}$, verificaram que a temperatura de secagem exerce influência sobre a velocidade de secagem do produto, sendo o tempo de secagem menor com o aumento da temperatura, com tempos de secagem iguais a 600, 162 e 138 min, respectivamente. Esse comportamento deve-se ao fato das maiores taxas de remoção de água do produto ocorrem nas maiores temperaturas, o que reduz o tempo de secagem.

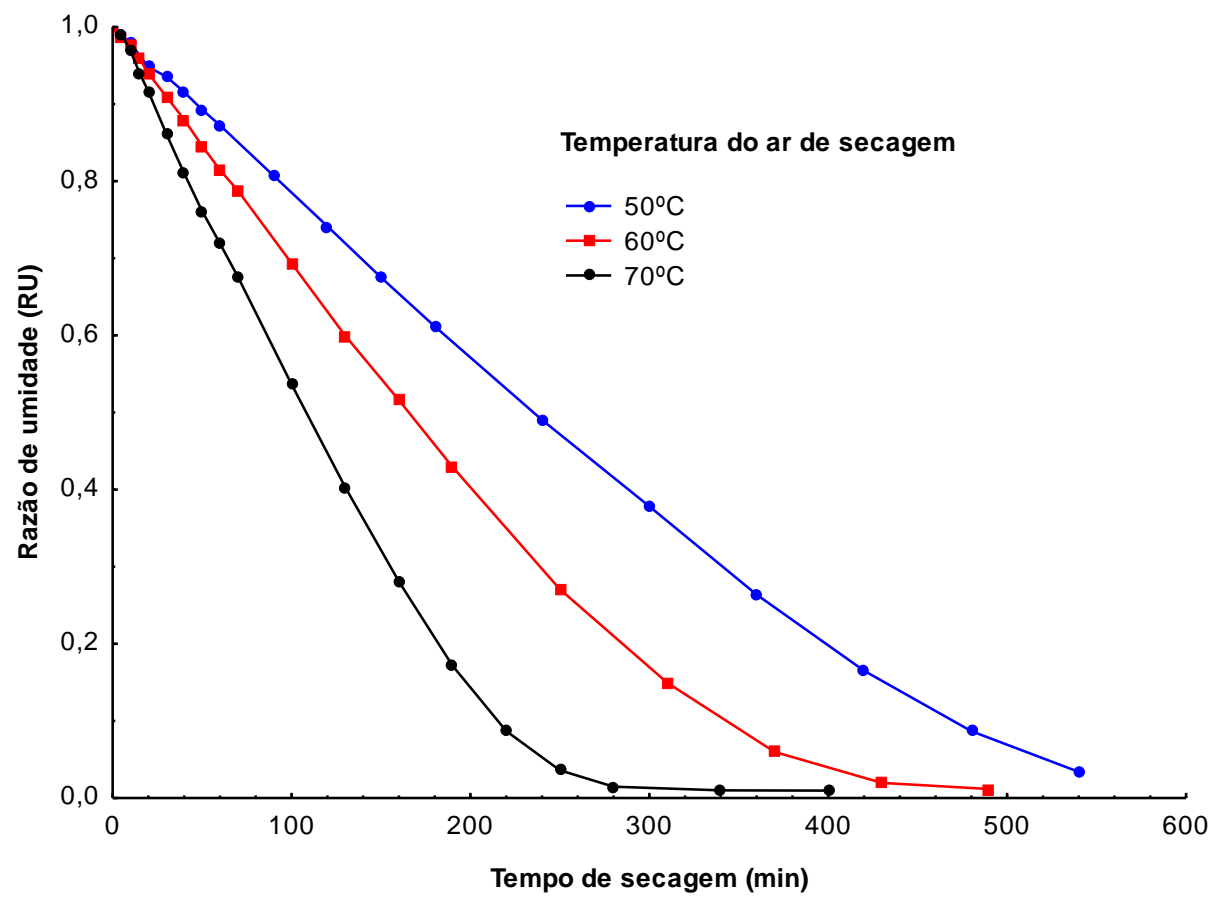

Figura 1. Cinéticas de secagem da pimenta de cheiro em diferentes temperaturas de secagem

$\mathrm{Na}$ Tabela 2 encontram-se os valores dos coeficientes de determinação (R2) e dos erros médios relativos (SE), calculados para verificação do ajuste dos modelos matemáticos aos dados experimentais da secagem da pimenta nas temperaturas de 50,60 e $70^{\circ} \mathrm{C}$. Observa-se que a maioria dos modelos apresentou coeficientes de determinação (R2) superior a 0,98 e erro médio estimado (SE) menor que 0,07 , sendo considerado um ajuste satisfatório. Porém, dentre os modelos matemáticos analisados, o modelo de Midilli et al. foi o que se ajustou melhor aos dados experimentais, por apresentar coeficiente de determinação com valores superiores a 0,998 e valores de erro médio estimado (SE) menor que 0,02, para todas as temperaturas estudadas. Barbosa et al. (2011) e Darvishi et al. (2014), estudando a 
secagem de pimentas observaram que o modelo de Midilli et comparar com os outros modelos estudados. al. foi o que melhor se ajustou aos dados experimentais ao

Tabela 2 - Valores dos coeficientes de determinação (R2) e do erro médio estimado (SE) dos modelos matemáticos da secagem da pimenta de cheiro nas temperaturas de 50,60 e $70^{\circ} \mathrm{C}$

\begin{tabular}{|c|c|c|c|}
\hline $\begin{array}{l}\text { Modelos } \\
\text { Matemáticos }\end{array}$ & $\begin{array}{c}\text { Temperatura } \\
\left({ }^{\circ} \mathrm{C}\right)\end{array}$ & $\mathbf{R}^{2}$ & SE \\
\hline \multirow{3}{*}{ Page } & 50 & 0,9972 & 0,0233 \\
\hline & 60 & 0,9979 & 0,0220 \\
\hline & 70 & 0,9983 & 0,0232 \\
\hline \multirow{3}{*}{ Dois termos } & 50 & 0,9875 & 0,0535 \\
\hline & 60 & 0,9889 & 0,0585 \\
\hline & 70 & 0,9893 & 0,0620 \\
\hline \multirow{3}{*}{ Newton } & 50 & 0,9839 & 0,0547 \\
\hline & 60 & 0,9837 & 0,0626 \\
\hline & 70 & 0,9835 & 0,0702 \\
\hline \multirow{3}{*}{ Henderson e Pabis modificar } & 50 & 0,9875 & 0,0581 \\
\hline & 60 & 0,9880 & 0,0629 \\
\hline & 70 & 0,9893 & 0,0666 \\
\hline \multirow{3}{*}{ Henderson e Pabis } & 50 & 0,9875 & 0,0498 \\
\hline & 60 & 0,9880 & 0,0550 \\
\hline & 70 & 0,9893 & 0,0583 \\
\hline \multirow{3}{*}{ Logarítmico } & 50 & 0,9997 & 0,0068 \\
\hline & 60 & 0,9978 & 0,0249 \\
\hline & 70 & 0,9951 & 0,0380 \\
\hline \multirow{3}{*}{ Midilli et al. } & 50 & 0,9999 & 0,0044 \\
\hline & 60 & 0,9993 & 0,0124 \\
\hline & 70 & 0,9987 & 0,0210 \\
\hline \multirow{3}{*}{ Difusão três termos } & 50 & 0,9261 & 0,1156 \\
\hline & 60 & 0,9373 & 0,1247 \\
\hline & 70 & 0,9429 & 0,1292 \\
\hline \multirow{3}{*}{ Wong e Sing } & 50 & 0,9998 & 0,0061 \\
\hline & 60 & 0,9985 & 0,0185 \\
\hline & 70 & 0,9975 & 0,0271 \\
\hline
\end{tabular}

Na Tabela 3, observa-se os valores estimados dos parâmetros do modelo de Midilli et al. para a pimenta de cheiro em diferentes temperaturas de secagem. Observa-se que as constantes $\mathrm{n}$ e $\mathrm{b}$ aumentaram com o aumento da temperatura; enquanto a constante a e $\mathrm{k}$ diminuem com o aumento da temperatura do ar de secagem. Radünz et al. (2010) estudando a secagem da folha de sálvia nas temperaturas de 50,60 e $70^{\circ} \mathrm{C}$, também observaram que a magnitude da constante de secagem $(\mathrm{k})$, que representa o efeito das condições externas de secagem, diminuiu com a elevação da temperatura do ar de secagem; enquanto as constantes $\mathrm{n} \mathrm{e} \mathrm{b}$ aumentaram com o aumento da temperatura.

Tabela 3 - Valores dos coeficientes do modelo de Midilli et al. ajustados para a secagem da pimenta de cheiro em diferentes condições de temperatura do ar

\begin{tabular}{lcrc}
\hline & \multicolumn{3}{c}{ Modelos matemáticos } \\
\hline \multirow{2}{*}{ Parâmetros } & \multicolumn{3}{c}{ Midilli et al. } \\
\cline { 2 - 4 } & $50{ }^{\circ} \mathrm{C}$ & $60{ }^{\circ} \mathrm{C}$ & $70{ }^{\circ} \mathrm{C}$ \\
\hline $\mathrm{K}$ & 0,001140 & 0,000918 & 0,000763 \\
$\mathrm{~A}$ & 1,000852 & 0,993254 & 0,987480 \\
$\mathrm{~B}$ & $-0,000529$ & $-0,000179$ & $-0,000061$ \\
$\mathrm{~N}$ & 1,106080 & 1,287393 & 1,463779 \\
\hline
\end{tabular}

Tem-se na Figura 2 as curvas de secagem ajustada pelo modelo de Midilli et al. nas temperaturas de secagem de 50,
60 e $70^{\circ} \mathrm{C}$. Nota-se o bom ajuste deste modelo nas descrições dos resultados experimentais. 


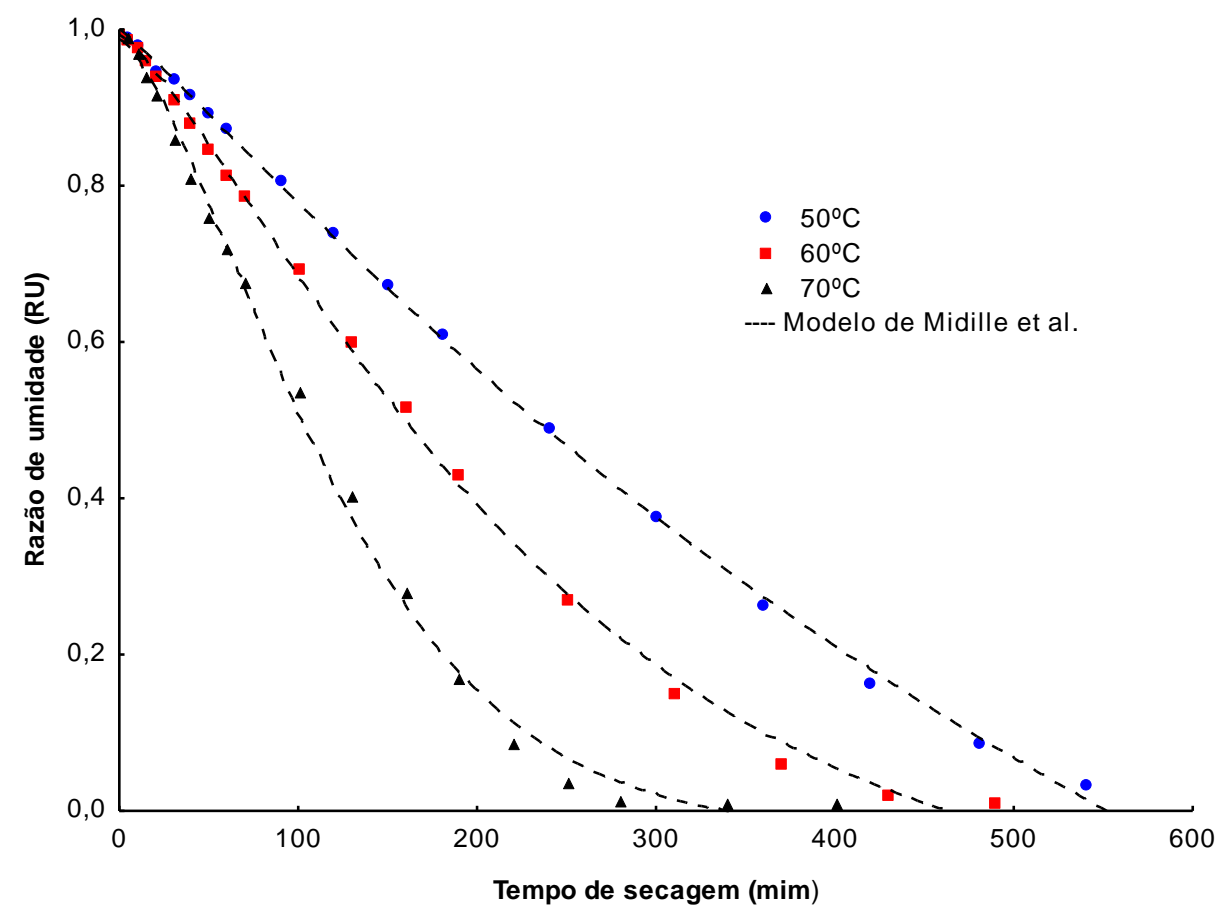

Figura 2. Curvas de secagem determinadas pelo modelo de Midilli et al. nas temperaturas de secagem de 50,60 e $70{ }^{\circ} \mathrm{C}$

Na Tabela 4 observam-se os valores de difusividade efetiva (D) obtidos para a pimenta de cheiro nas temperaturas de 50,60 e $70^{\circ} \mathrm{C}$. A equação utilizada foi baseada na Lei de Fick, com três termos para produtos com forma geométrica de placa plana, para calcular a difusividade efetiva do produto (D).

Verifica-se que os valores da difusividade efetivas aumentaram com a elevação da temperatura de secagem, variando de $8,900 \times 10^{-10}$ a $2,293 \times 10^{-9}$, apresentando o mesmo comportamento relatado por Reis et al. (2011). Verificando a diminuição das resistências internas de secagem com o aumento da temperatura. De acordo com Zogzas et al. (1996), os valores da difusividade efetiva (D) para produtos alimentícios se encontram na faixa de $10^{-11}$ a $10^{-9} \mathrm{~m}^{2} \mathrm{~s}^{-1}$. Observando-se que a pimenta de cheiro encontrase dentro desses valores.

Tabela 4 - Valores da difusividade efetiva (D) obtidos para a pimenta de cheiro em diferentes temperaturas de secagem

\begin{tabular}{cc}
\hline Temperatura $\left({ }^{\circ} \mathrm{C}\right)$ & Difusividade $\left(\mathrm{m}^{2} \mathrm{~s}^{-1}\right)$ \\
\hline 50 & $8,900 \times 10^{-10}$ \\
60 & $1,384 \times 10^{-9}$ \\
70 & $2,293 \times 10^{-9}$ \\
\hline
\end{tabular}

A Figura 3 encontram-se os valores de $\mathrm{D}$ a uniformidade de variação da difusividade com a apresentados na forma de "ln D" descritos em função do temperatura.. recíproco da temperatura absoluta $(1 / \mathrm{T})$. A reta obtida indica

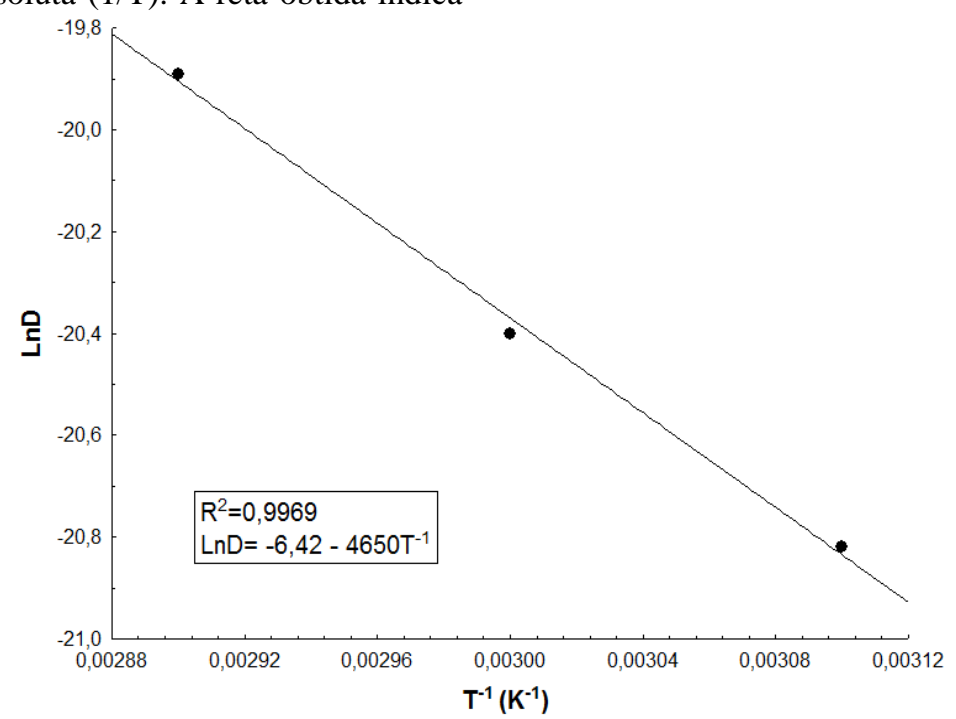

Figura 3. Representação de Arrhenius para a relação entre a difusividade efetiva e a temperatura absoluta na secagem da pimenta de cheiro 
A energia de ativação $\left(E_{a}\right)$ calculada da reta obtida na Figura 3, foi de 38,66 $\mathrm{kJ} \mathrm{mol}^{-1}$, encontrando-se na faixa de valores apresentado por Derlan et al. (2013) para a pimenta Cambuci nas temperaturas de 40,50 e $60^{\circ} \mathrm{C}$, que foi de 50,41 $\mathrm{kJ} . \mathrm{mol}^{-1}$ para a pimenta em pasta e de $32,86 \mathrm{~kJ} \mathrm{~mol}^{-1}$ para a pimenta picada. Nos processos de secagem, quanto menor a energia de ativação maior será a difusividade de água no produto (GONELI, 2007).

\section{CONCLUSÕES}

O tempo de secagem da pimenta de cheiro diminui com o aumento da temperatura do ar de secagem de 50,60 e $70{ }^{\circ} \mathrm{C}$, respectivamente. A maioria dos modelos matemáticos representaram satisfatoriamente os dados experimentais. Porém, o modelo Midilli et al. foi o que melhor se ajustou aos dados experimentais. Os valores do coeficiente de difusão aumentaram de acordo com a elevação da temperatura do ar de secagem, apresentando resultados entre 8,900 × $10^{-10}$ a $2,293 \times 10^{-9} \mathrm{~m}^{2} \mathrm{~s}^{-1}$. O valor da energia de ativação foi de $38,66 \mathrm{~kJ} \mathrm{~mol}^{-1}$.

\section{REFERÊNCIAS BIBLIOGRÁFICAS}

AFONSO JÚNIOR, P. C.; CORRÊA, P. C. Comparação de modelos matemáticos para descrição da cinética de secagem em camada fina de sementes de feijão. R. Bras. Eng. Agríc. Ambient., v.3, n.3, p.349-53, 1999.

ARAÚJO, E. I. M.; FREIRE, L. C. C.; ALVES, L. A.; BERTINI, L. M. Caracterização fitoquímica e atividade antioxidante dos extratos em etanol de Capsicumchinense (Pimenta de Cheiro). In: Congresso de iniciação científica do IFRN, 9, 2013. p. 597-603.

BARBOSA, F. F.; MELO, E. C.; SANTOS, R. H. S.; ROCHA, R. P.; MARTINAZZO, A. P.; RADÜNZ, L. L.; GRACIA, L. M. N. Evaluation of mathematical models for prediction of thin-layer drying of Brazilian lemonscented verbena leaves (Lippia alba (Mill) n.e. Brown). Rev. bras. prod. Agroind., v.9, n.1, p.73-82, 2007.

CERQUEIRA, P. A. Conservação pós colheita de pimentas de cheiro (capsicun chinense) armazenadas sob atmosfera modificada e refrigeração. Universidade de Tocantins, Gurupi-GO, 4.p, 2006. (Tese de Mestrado)

DARVISHI, H.; ASL, A. R.; AGHARI, A.; AZADBAKHT, M.; NAJAFI, G.; KHODAEI, J. Study of the drying kinetics of pepper. Journal of the Saudi Society of Agricultural Sciences, v. 13, n. 2, p. 130-138, 2014.

DERLAN, J. M.; SILVA, F. S.; PORTO, A. G.; LEITE, A. L. M. P.; PASTRO, D. C. Análise da cinética de secagem de pimenta Cambuci em diferentes temperaturas e formas de processamento. R. Encicl. Biosf., v.9, n.17, p. 97-107, 2013.

DUTRA, F. L. A; BRANCO, I. G.; MADRONA, G. S.; HAMINIUK, C. W. I. Avaliação sensorial e influência do tratamento térmico no teor de ácido ascórbico de sorvete de pimenta. Revista Brasileira de Tecnologia Agroindustrial, v. 04, n. 02: p. 243-251, 2010.

EMBRAPA - Empresa Brasileira de Pesquisa Agropecuária. Sistema de produção. Pimenta (Capsicum spp.). Disponível em: http://sistemasdeproducao.cnptia.embrapa.br/FontesHTM L/Pimenta/Pimenta_capsicum_spp/importanciaeconomica .html. 20 de abril de 2014 .

GAVA, J. A. Princípios da Tecnologia de Alimentos, São Paulo: Nobel, 1984.

GONELI, A. L. D. Dinâmica da variação das propriedades físicomecânicas e da qualidade das sementes de mamona (Ricinus communis L.) durante a secagem e o armazenamento sob condições controladas. Viçosa: UFV, 2007. 192p. (Tese Doutorado).

PONTES, S. F. O.; SANTOS, C. T.; FERREIRA, R. C; PONTES, V. L; FONTAN, R. C. I. Determinação das curvas de secagem em camada delgada de pimenta de cheiro (capsicun chinense) a diferentes temperaturas. Rev. bras. prod. Agroind., v. 11, n. 2, p. 143-148, 2009.

RADUNZ, L. L.; MOSSI, A. J.; ZAKRZEVSKI, C. A.; AMARAL, A. S.; GRASSMANN, L. Drying kinetics analysis of sage leaves. R. Bras. Eng. Agríc. Ambient., v. 14, n. 9, p. 979-986, 2010.

REIS, R. C.; BARBOSA, L. S.; LIMA, M. L.; REIS, J. S.; DEVILLA, I. A.; ASCHERI, D. P. R. Mathematical modeling of drying kinetics of pepper Cumari do Para. R. Bras. Eng. Agríc. Ambient., v. 15, n. 4, p. 347-353, 2011.

RESENDE, O.; FERREIRA, L. U.; ALMEIDA, D. P. Modelagem matemática para descrição da cinética de secagem do feijão Adzuki (Vigna angularis). R. Bras. Eng. Agríc. Ambient., v. 12, n. 2, p. 171-178, 2010.

SANTOS, B. A. J.; SILVA, F. G.; PAGANI, C. A. A. Estudo Cinético de secagem da pimenta malagueta (capsicun spp) cultivado no estado de Sergipe. Rev. GEINTEC, v. 2, n. 5, p. 465-471, 2012.

ZOGZAS, N. P.; MAUROULIS, Z. B.; MARINOS-KOURIS, D. Moisture diffusivity data compilation in foodstuffs. Drying Technology, v. 14, p. 2225-2253, 1996. 